\title{
Test particle acceleration in a numerical MHD experiment of an anemone jet ${ }^{\star}$
}

\author{
K. J. Rosdahl $1^{1,2}$ and K. Galsgaard ${ }^{2}$ \\ 1 CRAL - Observatoire de Lyon, 9, avenue Charles Andre, 69561 Saint Genis Laval Cedex, France \\ 2 Niels Bohr Institute, Blegdamsvej 17, 2100 Copenhagen Ø, Denmark \\ e-mail: kg@astro.ku.dk
}

Received 23 October 2009 / Accepted 23 December 2009

\section{ABSTRACT}

\begin{abstract}
Aims. To use a 3D numerical MHD experiment representing magnetic flux emerging into an open field region as a background field for tracing charged particles. The interaction between the two flux systems generates a localised current sheet where MHD reconnection takes place. We investigate how efficiently the reconnection region accelerates charged particles and what kind of energy distribution they acquire.

Methods. The particle tracing is done numerically using the Guiding Center Approximation on individual data sets from the numerical MHD experiment.

Results. We derive particle and implied photon distribution functions having power law forms, and look at the impact patterns of particles hitting the photosphere. We find that particles reach energies far in excess of those seen in observations of solar flares. However the structure of the impact region in the photosphere gives a good representation of the topological structure of the magnetic field.
\end{abstract}

Key words. acceleration of particles - magnetic fields - magnetohydrodynamics (MHD) - Sun: corona

\section{Introduction}

To investigate the dynamical evolution of the large scale phenomena seen in the solar atmosphere, one traditionally adopts the magneto-hydrodynamic (MHD) representation. The MHD approach is a macroscopic approximation describing the time dependent evolution of a plasma. The assumption when adopting MHD is that the time dependent plasma evolution is well described by macroscopic parameters, such as temperature, density, bulk velocity and magnetic field. This requirement is fulfilled when the plasma is in a local thermodynamic equilibrium and the characteristic MHD length scale is much longer than the mean free path of the plasma particles. For most of the time the plasma in the lower solar atmosphere obeys this requirement, but there are special situations for which MHD is not a good representation. One such situation is magnetic reconnection. Despite this fact, magnetic reconnection has for many decades been investigated using the MHD approximation (See Priest \& Forbes 2000, for a review.). In MHD the non-ideal evolution imposed by viscosity and resistivity in the plasma is described through a parameterization that approximates the real particle interaction on length scales well below the typical resolution of MHD. The choice of the form and value of the magnetic resistivity, $\eta$, naturally has implications on how the reconnection process progresses.

From the vast literature on 2D MHD reconnection, a common feature is the presence of a localised diffusion region, from which standing slow mode shocks extend along the separator lines - the Petschek like picture (Petschek 1964). When redoing this type of experiments with particle codes, one finds a

$\star$ Three movies are only available in electronic form at http: //www . aanda.org different picture. The existence of the MHD slow shocks vanishes as the evolution of protons and electrons decouples on sufficiently short length scales removing the plasma's ability to make a single coherent shock structure (Birn \& Priest 2007, See the article by Drake and Shay). Particle simulations allow for distribution functions to reach significant deviations from Maxwell distributions, typically providing a power law tail of high energy particles accelerated away from the diffusion region. One could therefore fear that the global evolution of the field will be qualitatively different depending on which method one chooses to use for the investigation. Recent comparisons between different approximations, going from MHD to particle in cell (PIC), in a controlled reconnection experiment have shown that despite variations in the reconnection speed between the different approaches, the same amount of flux reconnects and the same general structure of the final magnetic field results (Birn et al. 2005). Numerical MHD experiments therefore do capture the large scale dynamics of the evolution, and will at worst provide a somewhat wrong time scale and energetics for the dynamical evolution.

In the Solar atmosphere magnetic reconnection is deemed responsible for various explosive events, and the conversion of magnetic energy into other forms is therefore an important process one needs to understand to be able to explain observations. In the MHD approximation magnetic energy is converted to bulk motion and Joule heating of the plasma. This is a limitation, that already became clear from observations of X-rays and $\gamma$-rays in the late 1970's. Already then it was found that a significant amount of the energy release associated with large flares is converted into energetic particles (10-50\%) (Lin \& Hudson 1976; Lin et al. 2003). The effect of the accelerated particles is seen in different characteristic features. In the general picture one 
assumes that reconnection takes place in the corona, and that the strong electric field parallel to the magnetic field accelerates electrons and protons to velocities where their cross section becomes so small that they interact very little with the coronal plasma. Only when they reach the dense plasma close to the photosphere are they stopped very efficiently while, causing them to emit bremsstrahlung radiation. In loop flares, this gives rise to $\mathrm{H}_{\alpha}$ ribbons in the photosphere that move apart with time. The combined information of the motion and the magnetic field distribution in the photosphere provides indirect information about the reconnection speed in the acceleration region (Hoyng et al. 1981; Isobe et al. 2002; Jing et al. 2007; Liu et al. 2008a; Liu \& Wang 2009). In the reconnection model, one also expects the particles to be accelerated along the open field lines reaching into the upper corona. Hard X-ray observations have been made at the loop top of flares (Masuda et al. 1994), but it is not until recently that RHESSI has been able to detect a signature of independent particle distributions on either side of an assumed current sheet (Krucker et al. 2008). This investigation strongly supports the picture where the acceleration region is located in the corona, and that particles are accelerated in both directions away from the local reconnection region. Their analysis also indicate that the very energetic particles can be maintained in the local vicinity of the diffusion region long enough to be able to interact with the local plasma before continuing away from this region. Understanding the particle acceleration and interaction process is therefore important for understanding the distribution of the released magnetic energy in the flare process.

The lack of information regarding particle acceleration in numerical MHD experiments has led us to start investigating its effect. We adopt a simplified approach, where test particles are traced in snapshots from a numerical MHD experiment. The aim is to investigate the efficiency of the acceleration through forming distribution functions, looking at the patterns they form as they exit the allowed tracing domain and especially calculate radiation spectra from the bremsstrahlung that arises when the fast electrons are stopped in the top layer of the dense photosphere.

The layout of the paper is as follows. In the following section we review the relevant information about the numerical MHD experiment used as background field for test particle tracing. Section 3 describes the numerical approach used for tracing the particles, while Sect. 4 gives the setup of the tracing experiment. In Sect. 5, the results of the data analysis are presented. Section 6 discusses the drawbacks with the approach and what we need to do to improve the results, while Sect. 7 draws up the conclusions of the paper and suggests further work to be done.

\section{Numerical MHD experiment}

The dynamical evolution of an emerging flux tube entering into an open coronal field was investigated using a numerical 3D MHD approach by Moreno-Insertis et al. (2008). The numerical experiment consists of a stratified atmosphere covering the region from $4 \mathrm{Mm}$ below the photosphere to $29 \mathrm{Mm}$ above it. Imbedded in the convection zone is a twisted flux tube that over a fraction of its length is made buoyant. Additionally a slanted magnetic field permeates the numerical domain, representing an open magnetic field configuration. The part of the flux tube that rises from the convection zone pushes away the slanted magnetic field to make space for itself. While the tube is still in the convection zone, the motions are slow and not very compressible, and very little interaction between the two flux systems takes place. As the rising tube reaches the photosphere the magnetic field strength starts to build up. Shortly after, the vertical magnetic pressure gradient becomes sufficient to initiate the emergence of the flux tube into the upper atmosphere. The emergence speed in this phase is much higher than the slow rise speed observed in the convection zones. The initial plasma $\beta$ decreases with height through the transition region and into the corona making it easier for the two magnetic flux systems to come into close contact. The change in $\beta$ is important for the dynamical evolution of the boundary layer between the two flux systems, especially where they experience large differences in field line orientation. Such regions are prone to generating strong current concentrations as they are compressed by the emergence process. As part of the flux tube emerges into the corona a current dome separating the two flux systems is formed. The continued emergence concentrates the current in the dome into a long thin sheet connecting along the length of the emerged flux, laying skew to one side of the summit line of the emerged flux. Eventually the current density becomes large enough for non-ideal MHD processes to start changing the field line connectivity. The reconnection between the magnetic field from the rising loop and the ambient open field creates a new low lying loop system next to the emerging one and new field lines that connect the deeper parts of the twisted loop with the open flux. The effect of reconnection is therefore to eat away the emerging flux and build up a secondary loop system. One way to see this is to investigate how the separatrix surface between the two flux systems changes with time. This is investigated by Galsgaard \& Moreno-Insertis (2010, in prep.) and shows how the interaction between the magnetic field and the photosphere changes structurally with time, clearly indicating the continued diminishing of the area occupied by the emerging loop system and the concurrent growth of the new loop system. Figure 1 shows the connectivity domains at three different times during the emergence process. The background shading represents the photospheric magnetogram indicating the sign and magnitude of the magnetic fields normal component. This shows a bipolar structure imbedded in a monopolar background field. From the magnetogram alone one could be led to conclude that a new bipolar field has emerged into the photosphere with no complicated connectivity pattern. This is not the case: The lines on the map show the intersection of the separatrix surfaces with the photosphere. The main features are the outer line defining the perimeter inclosing flux that connects both ends to the photosphere. A line dividing the perimeter represents the division between the two loop systems, where the top one is the secondary loop created by the magnetic reconnection process. The jagged form of the outer line is created by convective motions that overshoot above the plane in which this surface is shown.

As a consequence of the changes in magnetic connectivity, two reconnection jets are accelerated out of the current sheet edges. The most prominent of the jets escapes upwards, giving rise to high velocity plasma motions that follow the open field lines away from the solar surface.

The evolution of the numerical MHD experiment resembles the anemone jets which Hinode observations have found to be very common in open flux regions (Shibata et al. 2007).

The MHD approximation assumes the plasma to be in a statistical equilibrium, defined by frequent collisions between particles, where physical variables like temperature and bulk velocity are represent for the average particle in a small volume. Therefore, numerical MHD experiments are not able to provide indications as to how individual particles may react to strong electric fields parallel to the magnetic field. In a real plasma, this electric field component is responsible for accelerating a small fraction of particles up to relativistic velocities. As only a small fraction of the particles are accelerated, they give rise to 

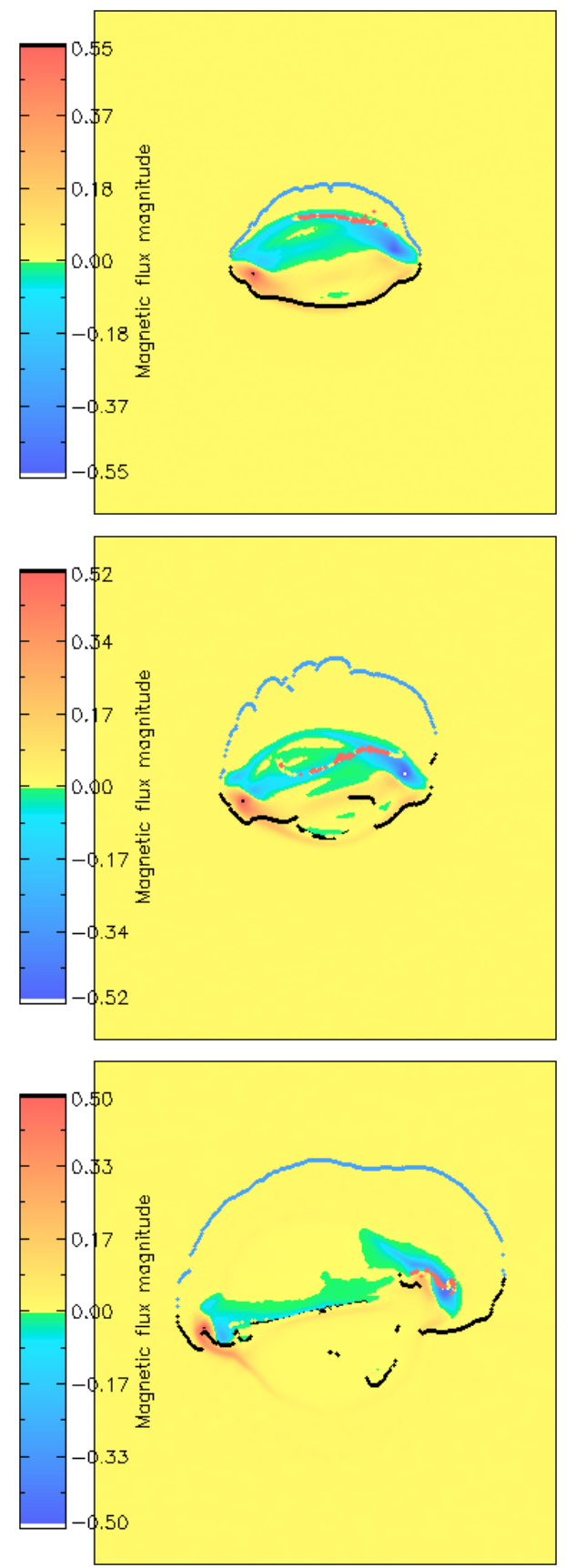

Fig. 1. The connectivity pattern of the magnetic field in the emergence process at three different times. The colour indicates the field strength and sign of the normal component of the magnetic field with blue representing negative flux. Each frame is scaled to its own dynamical range. The lines indicate the intersection of the separatrix surfaces with the photosphere.

a high energy power law tail attached to the Maxwellian velocity distribution. Power law distributions created in eruptive events like solar flares are well documented by RHESSI observations (Liu et al. 2008b; Lin 2008; Liu \& Wang 2009). In fact, integrating the energy content of this tail indicates that a large fraction, up to 50\% (Lin \& Rhessi Team 2002; Lin et al. 2003), of the energy released in the flare process may go into accelerating particles. If we therefore want to understand in detail the effect of the flare processes it is important to take this additional physics into account. This is not an easy task, as it requires models to resolve particle interactions, while still maintaining the large scale structures responsible for the dynamical driving of the magnetic system. Resolving both the particle scale and the large structural scales requires unrealistically large numerical resolution. A simpler approach is to assume:

- the integration time for the particle experiment is much shorter than the time between MHD snapshots;

- the MHD snapshot is static in time;

- there is no back reaction on the MHD field from the particle motions.

The first assumption is fulfilled in the following analysis, while the two later assumptions are unrealistic. However, using these makes it possible to obtain results regarding (i) the particle distributions after acceleration, (ii) the locations where the acceleration takes place and (iii) where the accelerated particles end up as they are rapidly accelerated away from their initial positions. The details of the applied approximation for this approach are discussed in the following section.

\section{Test particle tracing approach}

The relativistic equation of motion for a charged particle in the presence of a Lorentz force is described by the Lorentz equation (LE):

$\frac{\mathrm{dp}}{\mathrm{d} t}=q(\boldsymbol{E}+\boldsymbol{v} \times \boldsymbol{B})$,

where $p=m \gamma v$ is the relativistic momentum, $q$ the particle charge, $\boldsymbol{E}$ and $\boldsymbol{B}$ the electric and magnetic field at the particle position, $\boldsymbol{v}$ is the particle velocity, $m$ is the rest mass of the particle and $\gamma$ is the Lorentz factor. This equation describes the orbiting motion of a charged particle around any magnetic field. Before going further into the discussion of the applications of this equation it is worth looking into the characteristic radius of the orbiting motion of a charged particle in a magnetic field. The Larmor radius of this circular orbit is

$R_{\mathrm{c}}=\frac{\gamma m v}{B q}$,

where $v$ is the speed. For the emergence experiment the characteristic coronal values are $B=10^{-2} T$ and the coronal temperature 1.2 MK. Assuming a Maxwell-Bolzman distribution for the particles gives typical electron velocity of roughly $10^{7} \mathrm{~m} / \mathrm{s}$ and a $\gamma=1$. Using these values in the equation above, we obtain a Larmor radius of order $10^{-3} \mathrm{~m}$. This is a typical value, but more extreme circumstances, may change it by up to 2 orders of magnitude. The relevant quantity to compare to this value is the resolution of the emergence experiment. In this case the box size is $34 \mathrm{Mm}$ and the grid resolution on the order of 320 grid points. This gives a typical grid resolution in the numerical domain of order $0.1 \mathrm{Mm}$. Comparing numbers it is seen that the orbiting motions typically take place on $10^{-7}$ of a grid cell. Obtaining the magnetic field with this spatial resolution, from a single precision dataset, implies that the magnetic field seen by a particle in a given orbit, will be constant across the orbit, and its orbit will only change due to changes in the magnetic field direction taking place over large distances measured in Larmor radii. Using Eq. (1) to track particle motions in the numerical MHD experiment will therefore require a huge amount of computing time, while providing information that can be obtained using a simpler approximation. Instead of using the Lorentz equation to determine the particle paths, we use the Guiding Center Approximation (GCA) (Northrop 1964). The GCA integrates the $\boldsymbol{B}$-parallel motion and the non-parallel of charged 
particles, while ignoring the orbital motion, but keeping track of the magnetic moment. Ignoring the orbital motion implies that the time step for this approach can be up to several orders of magnitude larger than for the Lorentz equations, which can save a huge amount of computing time and provide the same particle trajectories. The relativistic GCA equations of motion are:

$$
\begin{aligned}
\frac{\mathrm{d} u_{\|}}{\mathrm{d} t}= & \frac{q}{m} E_{\|}-\frac{\mu}{\gamma m B}(\boldsymbol{B} \cdot \nabla) B, \\
\frac{\mathrm{d} R}{\mathrm{~d} t}= & \frac{u_{\|}}{\gamma B} \boldsymbol{B}+\frac{\boldsymbol{E} \times \boldsymbol{B}}{B^{2}}+\frac{\mu}{\gamma q B^{2}} \boldsymbol{B} \times \nabla B \\
& +\frac{m u_{\|}^{2}}{\gamma q B^{4}} \boldsymbol{B} \times(\boldsymbol{B} \cdot \nabla) \boldsymbol{B}, \\
\mu= & \frac{m u_{\perp}^{2}}{2 B}=\text { constant }
\end{aligned}
$$

where the $\|$ refers to the component along the magnetic field, $R$ is the center coordinate of the particle orbit and $\perp_{\perp}$ refers to the component perpendicular to the magnetic field vector - here only the magnitude is interesting and not it's angle. These equations represent the momentum equation, the motion of the guiding center and the adiabatic invariance of the magnetic moment.

We solve these GCA equations numerically using a 5th order Cash-Karp Runge-Kutta (Press et al. 1992) method with adaptive time stepping. As the electric and magnetic fields are provided by the 3D numerical MHD experiment, these values are only known at the grid locations provided by the MHD experiment. Values at the actual particle positions are obtained using a Bi-cubic interpolation scheme.

The data from the MHD simulations is saved in dimensionless units, and are scaled to SI units before being used in the tracing code. The electric field is not saved in the MHD code, and one can use different methods to derive the resistive contribution from the saved data Numerical codes typically uses resistivity models at constant $\eta$, but with various forms of current dependent $\eta$ threshold, higher than 2 nd order current dependence typically 4 th order - to localise the effective $\eta$ towards the numerical resolution limit combined with a "shock" capturing approach to localise diffusion in current sheet regions as long as new magnetic flux is advected into the sheets. In this case we have used the algorithm that was used in the numerical MHD experiment. The MHD code uses a high order finite difference approach on staggered grids to solve the MHD equations. The high order approach (6th order derivatives and 5th order interpolations) requires the special approach with 4 th order dependence combined with the "shock" capturing mechanism to avoid/limit numerical ringing effects in the physical variables in the vicinity of regions where the magnetic field changes significantly over a few grid points. A description of the algorithm is given in Nordlund \& Galsgaard (1995).

\section{Particle setup}

Test particle tracing has previously been done in a number of different environments. Mostly these have consisted of situations representing MHD turbulence (Dmitruk et al. 2003, 2004; Dmitruk \& Matthaeus 2006; Turkmani et al. 2006) finding the accelerated particles to reach high velocities, having power law distributions with steep power indexes (Turkmani et al. 2006). The typical result is that the fastest particles reach energies much higher than found in solar observations.

Ambrosiano et al. (1988) showed, using a 2D reconnection experiments, that having a single large scale diffusion region

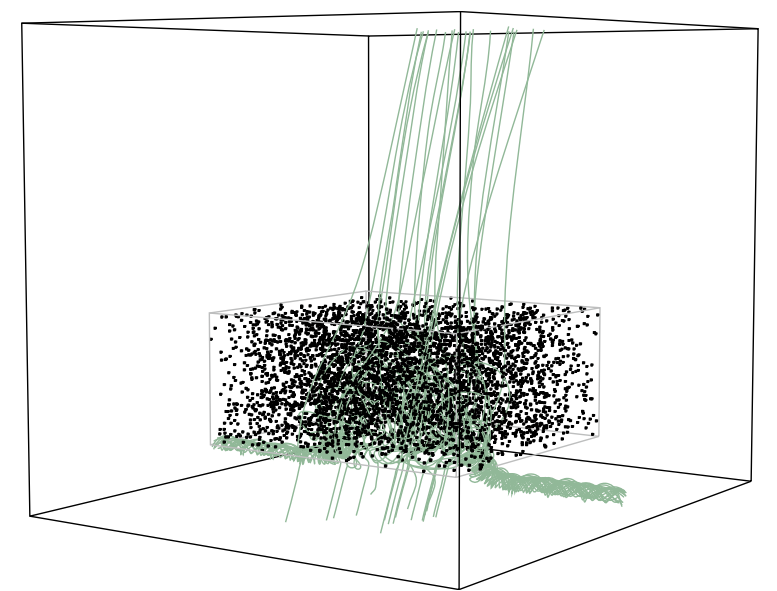

Fig. 2. The image represents the magnetic field line structure at one instance in the MHD simulations, showing the general structure of the magnetic field by tracing a number of representative magnetic field lines. The dots represent the starting positions of a smaller sample of the particles in the tracing experiments.

with internal structure gives rise to a stochastic acceleration process where initial close neighboring particles can experience very different accelerations histories as their paths diverge exponentially with time. This is clearly different from earlier experiments that consisted of laminar diffusion regions. The same process is seen in the previous 3D papers (Turkmani et al. 2006; Dmitruk et al. 2003, 2004; Dmitruk \& Matthaeus 2006), where, as a general concept, the whole numerical domain is in a turbulent state with acceleration sites scattered randomly throughout the numerical domain. In the present case we have a situation where the acceleration region is localised in the numerical domain, and the global topology of the magnetic field is not in a turbulent state as in the earlier mentioned 3D experiments. This does not imply that the diffusion region has a simple laminar structure, as it contains significant variations in both space and time and we therefore expect the acceleration process to be stochastic in nature. Therefore, it is interesting to investigate how particles are accelerated in this environment, which type of particle distribution arises, where they preferentially "exit" the free flight domain and which type of observational spectra are obtained from the bremsstrahlung generated as the particles impact with the photosphere.

To initiate the investigation, a sub volume of the MHD box is chosen to contain the traced particles. The lower boundary of this domain is the photosphere in the MHD model, below which the plasma density becomes so high that collisions will thermalize any accelerated particles almost instantaneously. The top boundary is chosen such that for all the snapshots in the numerical experiment it is above the current sheet region that is responsible for accelerating the particles. The extent along and across the loop is chosen to cover the maximum extent of the current sheet. A representation of the particle box seen in relation to the magnetic field configuration in the emergence phase is shown in Fig. 2.

We perform tracing analysis for all snapshots provided by the numerical MHD emergence experiment. For each of the 160 snapshots we initiate 90000 particles in the chosen sub domain. Inside this domain, they are given random positions and pitch angles. Finally they are assigned a velocity according to a Maxwellian distribution with a temperature of 1.2 MK. The particles are then traced for $0.5 \mathrm{~s}$ or until they either impact with the photosphere or exit the full MHD box above the photosphere. 
Tracing calculations are conducted for both protons and electrons. The results show that the parallel resistive electric field only provides for a small acceleration of the protons, while the much lighter electrons experience acceleration, even on multiple sites, that changes the distribution function significantly within the alloted tracing time. The following discussion will therefore only concern the results from the electron experiments.

\section{Results}

In this section we discuss the results obtained from test particle tracing in the static MHD snapshots. There are three objectives to this section. The first is to follow the particle trajectories to see where they end up after the $0.5 \mathrm{~s}$. As a fraction of these reach the photosphere, spatial distribution pattern indicates what we may expect to see in real observations, while the ones exiting the top boundary are expected to enter into the solar wind particle population. The second is to look at the velocity distribution functions obtained as a function of MHD snapshot to see how large a fraction of the particles are being accelerated and which energies they reach. Finally, we use the distribution function for the electrons that impact with the photosphere to derive spectral information that can be compared directly with observations.

\subsection{Impact patterns}

Following 90000 particle paths through the MHD domain is by no means feasible, but provides detailed insight to the individual reasons for particle acceleration. We will look at a few examples of this below. Apart from this, there are two other approaches we are going to investigate in this section. One is to follow a sample of particles as they are moving around the domain. The most interesting are the ones that impact either the photospheric or the top boundary. These give us an idea about the locations where we would expect to see secondary effects of the reconnection process, and allows us to obtain distribution functions and spectra of the impacting particles.

\subsubsection{Individual acceleration patterns}

In previous papers describing particle acceleration, it has been seen that the particles undergo repeated acceleration and deacceleration as they pass through the turbulent magnetic field domain (Dmitruk et al. 2004; Dmitruk \& Matthaeus 2006; Turkmani et al. 2006). In this experiment we assume that a single coherent current sheet is more likely to only provide one single acceleration of the particles, at least for the ones that very rapidly reach the domain boundaries. Figure 3 shows the time evolution of a number of electrons. This shows a time history that is similar to those seen in the previous papers, starting with a rapid acceleration followed by a period of cyclic behavior in kinetic energy. We can conclude that there are a number of particles that are trapped on trajectories where they repeatedly are moving forward and backward along the same magnetic field line. A small number of particles are seen to reach a peak energy level, which is subsequently represented by a line with constant energy. These are particles that have reached one of the imposed boundaries, from where further tracing is not possible. As can be seen, this type of particles reach the boundary rather fast, having experienced a rapid acceleration over a small fraction of a second.

From inspection of the animations of the particle motions (movies are available with the Web version of the paper) it

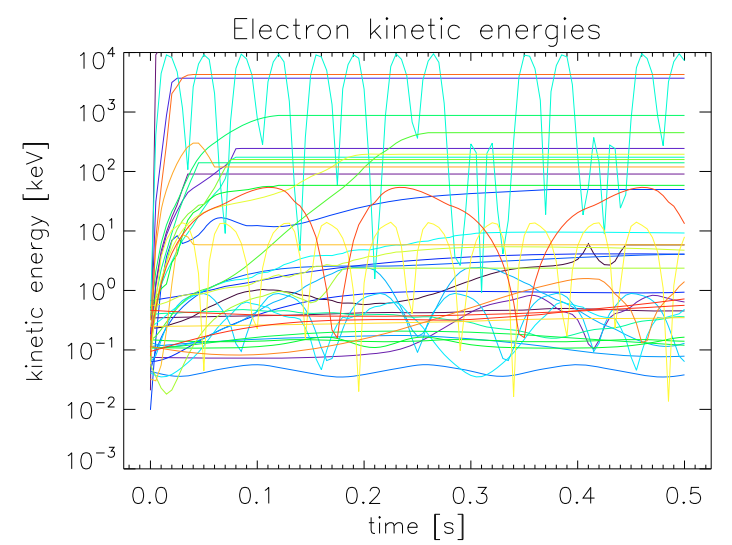

Fig. 3. The graph represents the kinetic energy of a small number of significantly accelerated particles as a function of time. The different colours represent different particles. The peak energy in the diagram, $10^{4} \mathrm{keV}$ does not encompass the most energetic particles in the experiment. It is noticed that all example particles are accelerated to energies many orders of magnitude above their thermal energies. About half of the sample particles stays at thermal energies.

is easy to see both the fast moving particles that exit the domain and the ones that seem to oscillate around an equilibrium position.

\subsubsection{The global view}

The behavior of the test particles depends strongly on the time of the snapshot from the numerical MHD experiment. Initially the particles are placed in a domain where no interaction between the two flux systems takes place. No parallel electric fields exist and the particles are just thermally moving along the magnetic field lines without significant changes in their energy.

As the flux tube starts to rise into the region where the particles are placed, effects on the particle behavior become visible. First the particles stay almost confined to the box in which they are initiated, but later they start moving around within this region gaining an increasing amount of energy. As the tube clearly emerges above the photosphere and a strong current sheet forms, more action is seen by a fraction of the particles. In Fig. 4 two snapshots are shown. Here the box represents the full MHD domain. The field lines indicate the structure of the magnetic field, while the colour of the particles indicates the energy obtained after $0.5 \mathrm{~s}$ of tracing. Looking at the images it is seen that only a small part of the volume becomes energized and that these particles are accelerated away from the reconnection region following the global structure of the magnetic field. What is not clear from these images is the shape of the particle energy distribution function as they are channeled into the photosphere, where they are assumed to be de-accelerated almost instantaneously, creating strong bremsstrahlung radiation (we will return to this below).

It is clear from the images in Fig. 4 that only a small fraction of the particles are exposed to a very strong acceleration (larger than $1 \mathrm{MeV}$ ). To make this clear, one can derive an energy distribution function after the $0.5 \mathrm{~s}$ integration. Doing this we ignore the fact that a large fraction of the particles that reach the defined boundaries do it well before the the $0.5 \mathrm{~s}$ has been reached. These are included as having the energy at the time of exit of the domain. Figure 5 shows the time evolution of the kinetic energy distribution function. Initially we see the Maxwell distribution. As time in the numerical MHD experiment progresses 
A\&A 511, A73 (2010)
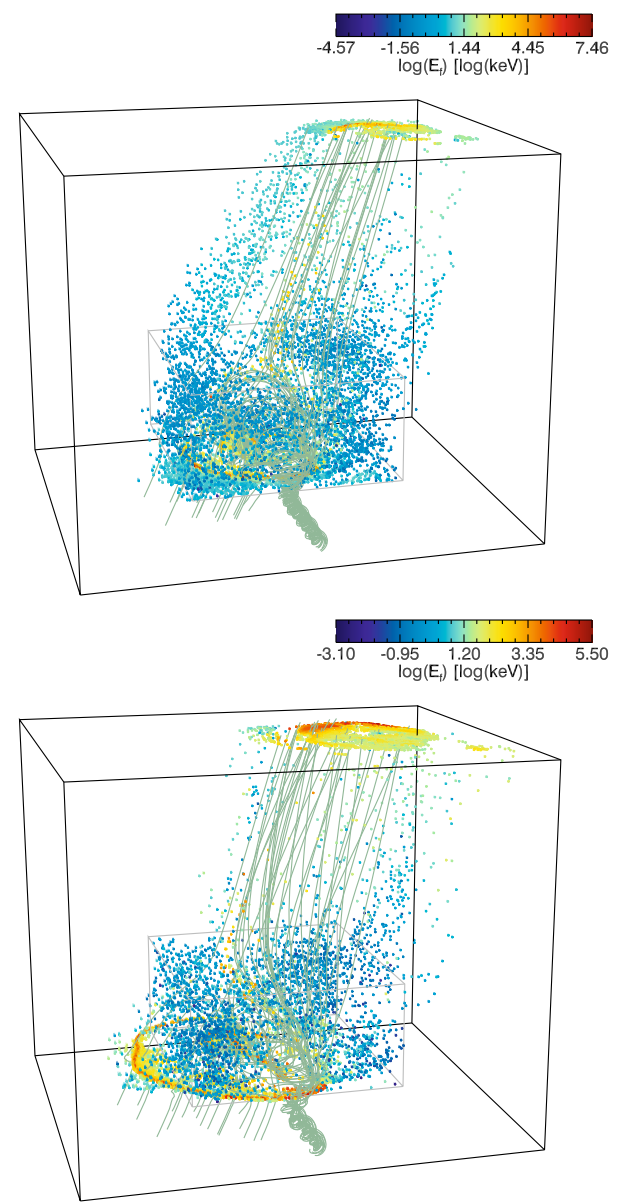

Fig. 4. The frames show the large MHD domain, with the imposed particles at their final positions for two different times in the numerical MHD experiment. The field lines outline the general structure of the magnetic field. The particles are colored relative to their final kinetic energy.

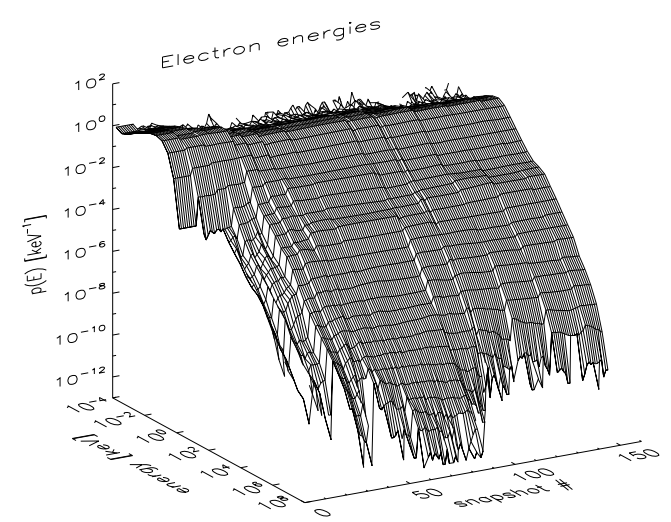

Fig. 5. The figure shows the time evolution of the particle kinetic energy distribution as a function of time in the numerical MHD experiment.

a clear high-energy tail develops. Only minor adjustments occur between the different MHD snapshots. The power slope of the distribution function is roughly maintained, while the peak energy changes, dependent on the level of activity in the snapshot. Looking at a single distribution function one can see different power slopes along the distribution, and it is possible to identify three different power indexes.

The peak energy reached in the experiment is orders of magnitude larger than observed in solar flares. This same observation is noticed in a previous analysis (Turkmani et al. 2006). We will return to this in the discussion below.

\subsubsection{Impact structures}

Direct observations of particle paths in the solar atmosphere is not possible. What is observed is the effect as the particles impact with the dense plasma in the photosphere. The radiation indicates where the field lines on which the accelerated particles move intersect with the photosphere. From the tracing experiments it is simple to make images showing where and with which energy the particles exit the allowed tracing domain. The most direct comparison can be made in the photosphere, were fast particles will loose their kinetic energy in exchange for heating the local plasma in a fraction of the observation time. This gives rise to local hot spots seen as $\mathrm{H}_{\alpha}$ ribbons in flares. We plot the positions and energies of the particles that reach the photosphere in Fig. 6. The frames show three snapshots representing different times in the numerical MHD experiment. The colour represents the energies of the particles impacting with the photosphere at any time in the $0.5 \mathrm{~s}$ integration time. The numbers in the lower left of the frames indicate the number of particles (out of the total number) that impact with the photosphere. The frames show a number of interesting features. In the early phases only few and low energy particles impact. As the reconnection sets in a characteristic pattern develops, an almost closed envelope with a thick finger running down through part of the region. What does this signature express? And why do the later structures show much more complexity at especially one of the outer edges? These questions need to be answered in order to better understand the acceleration process.

From the topology analysis of the numerical MHD experiment (see Galsgaard \& Moreno-Insertis, in prep., for details) it is found that the reconnection process creates three independent flux domains. One represents the initially open magnetic field region, one confines the emerging magnetic flux and a final region represents the new loop system created by the ongoing magnetic reconnection process. Figure 1 above shows images of how the connectivity pattern of the numerical MHD experiment changes with time in the photosphere, as already discussed.

Comparing Figs. 1 and 6 reveals close similarities between the patterns of the two analyses. This is expected, as the region where particles are accelerated coincides with the location of the intersection of the separatrix surfaces. On one hand the separatrix is a mathematical surface defining the boundary between two flux regimes. The intersection of the separatrix surface with the photosphere therefore defines a mathematical line. On the other hand, the current sheet, and the associated diffusion region, has a finite spatial extension. The field lines penetrating the diffusion region therefore cover a much larger area when traced down to the photosphere. The spatial extent of the accelerated particles, when impacting with the photosphere, must have a finite extent centered on the separatrix surfaces intersection with the photosphere.

The structure and location of the particle impact with the photosphere is therefore a direct consequence of the extent and mapping for field lines penetrating the diffusion region and connecting to the photosphere. But, why does it include these small structural variations seen in the last of the images? Looking at the same type of impact map further up in the atmosphere, the mapping contains a much simpler mapping structure. The reason for the complicated patterns is located in the lower parts of the atmosphere. Here the magnetic fields lines are found to bend in different directions due to a weak convection like motion 
K. J. Rosdahl and K. Galsgaard: Particle acceleration in a numerical MHD experiment
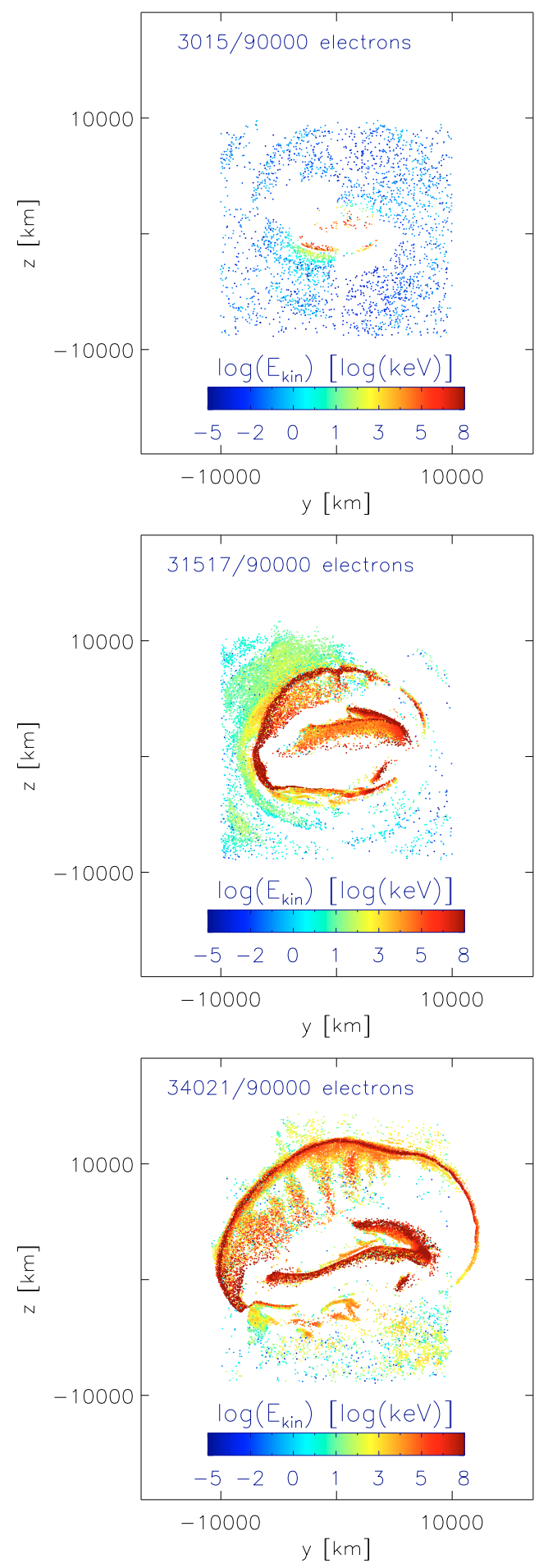

Fig. 6. The pattern and energy distribution of the particles after $0.5 \mathrm{~s}$ time integration. The colour indicates the particle kinetic energy on impact with the photosphere. The three images represent different times in the numerical MHD experiment.

taking place. This indicates that the pressure gradient locally dominates the momentum equation, implying that the plasma density may be high enough to thermalize the accelerated particles at this height in the atmosphere.

From comparing the images in the two figures it is clear that the impact region maps directly back to the acceleration region. Being able to make realistic field extrapolations from magnetograms into the corona, will therefore give a clear indication of

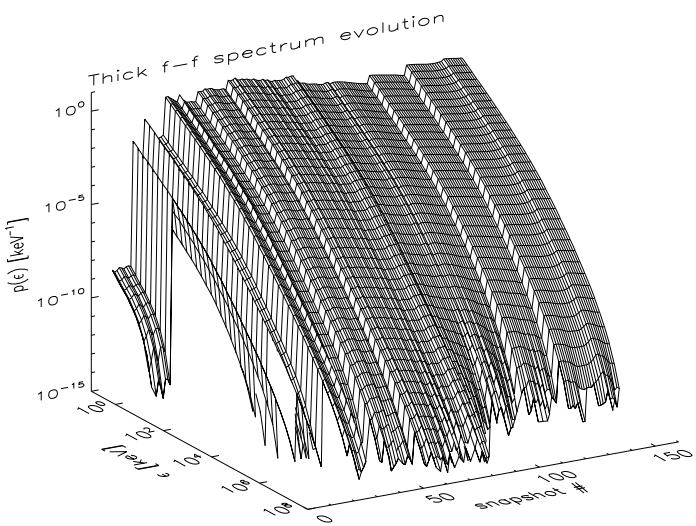

Fig. 7. The spectra obtained directly from the electron kinetic energy distribution functions for the electrons impacting with the photosphere in the tracing experiments. The assumption is that the particles are thermalised over a short timescale (compared with the observation time) and distance through bremsstrahlung.

where the diffusion region will be located in 3D space. Doing this with large precision is not a simple task in a magnetic field that is far from being in a potential or even linear force free state.

\subsection{Radiation spectra from the photospheric impact}

From a physical point of view the distribution functions give direct insight to the behavior of the particles. But, it is not a quantity that can be easily observed. Instead, we observe spectra from the radiation particles emit as they are de-accelerated on impact with the photosphere. This process occurs almost instantaneously which allows us to calculate the spectra directly from the particle distribution function. Brown (1971) and Holman (2003) provide detailed information about how to do this. Because of the very high energy tail of the distribution function, the relativistic formulae for the derivation must be used. This limits the energy of the most energetic photons in the spectrum, as it would otherwise only show one power law extending to very high energies.

The spectra obtained from this exercise is shown in Fig. 7. This shows two situations, both containing three different power law spectra depending on the energy range of the spectrum. The slope of the spectrum, covering the X-ray to gamma-ray range, changes from about 1.15 and 1.90. These slopes represent much harder spectra than observed from flare events. For large flares, the peak energy typically reaches hundreds of $\mathrm{MeV}$ and the $\mathrm{X}$-ray power index is typically in the range of 2.6 to 3.9 (Lin 2008).

\section{Discussion}

The previous section discusses the results we have obtained tracing test particles using MHD snapshots from a numerical flux emergence experiment. We have found that even in a situation where there is only one large current sheet located inside the numerical domain, is it possible to accelerate particles away from the initially given Maxwell distribution. The final result is an electron distribution function that contains a high energy power law tail which extends from the Maxwell distribution. After the onset of MHD reconnection, this distribution forms rapidly and maintains a similar structure throughout, although the peak energy and fraction of accelerated particles varies with time. 
There are a number of critical issues related to the approach used here:

- Individual particles follow paths that gyrate around magnetic field lines. As part of the analysis we have compared the results obtained using both the correct Lorentz equation for the particle motion and the much faster GCA. It is clear that for this experiment, the two methods produce almost identical results. The reason for this comes from the fact that the background field from the numerical MHD experiment does not have a grid resolution that is even close to the Larmor radius of the particle orbits. The magnetic and electric field therefore have spatial variations on length scales that are many orders of magnitude larger, which makes the GCA a good description of the particle motion. There is therefore no reason to use the LE description as long as the numerical MHD experiment describes phenomena on typical solar surface length scales.

- A limitation using the test particle tracing is the lack of interaction between the particles present in the domain. Each particle is treated independently of all other particles in the domain. This has a number of problems:

- There are no built in collisions, which allows particles to be accelerated far more than in a realistic plasma.

- There is no electric interaction, implying that the charge separation between the electrons and the much slower protons is again unrealistic.

- Even if we included simple particle collisions, then a number of particles would reach high energies and their collision cross sections would decrease sufficiently for them to escape the acceleration region. But, as the particles are accelerated, there is a natural exchange of energy with the macroscopic field. This would decrease the E-field in the diffusion region and the remaining particles would feel a smaller acceleration force, making it less likely that they would experience the same significant acceleration and escape from the region.

- The above items have significant implications on the obtained distribution functions. In the present approach too many particles reach far too high energies. The lack of back reaction on the macroscopic fields allows the production of power laws that are too hard compared with observations. These problems naturally influence the derived bremsstrahlung spectrum generated by the particles impacting with the photosphere.

To improve on these issues we need a different and much more calculation heavy approach. One way is to adopt a PIC approach, where one assumes the included particles to have a very exaggerated weight to allow the domain to cover the MHD scales without being dependent on resolving spatially the Larmor radii of real charged particles. This approach is being investigated.

\section{Conclusions}

The usage of test particle tracking in MHD simulations constitutes a simple way to obtain information about regions of particle acceleration and the spectra and spatial distribution on planes of interest. When the MHD data represents a large solar structure and the typical length scale involved is much larger than the particle Larmor radius, the Guiding Center Approximation is the favored method to obtain these results. Comparisons between the GCA and the full Lorentz solution for this data discussed in this paper show no differences in results, while on the computational side the GCA is between a 100 and a 1000 time cheaper in computing time.

Warnings must be given. The lack of back reactions between the accelerated particles and the provided MHD field results in far too many particles being accelerated to far too high energies. The distribution functions and spectra obtained from such calculations are therefore giving too many high energy particles resulting in too hard spectra when calculating the bremsstrahlung emitted by the particles interacting with the dense photosphere.

On the positive side, it is possible to use the impact location on the photosphere to identify the location of the separator surfaces connecting up to the diffusion region where the acceleration is taking place. This information may be a help to guide magnetic field extrapolations of the photospheric field distribution, as one can both see the impact points in the photosphere and the X-ray region in the corona where the reconnection process takes place. These have to fit with the topological structure obtained from the extrapolation models.

To obtain more generally useful information from this type of exercise one has to adopt a more physically consistent model, which takes the whole approach into a much more complicated problem. But one we have to find a solvable solution to if we want to obtain reliable information from numerical experiments.

\section{References}

Ambrosiano, J., Matthaeus, W. H., Goldstein, M. L., \& Plante, D. 1988, J. Geophys. Res., 93, 14383

Birn, J., \& Priest, E. R. 2007, Reconnection of magnetic fields: magnetohydrodynamics and collisionless theory and observations, ed. J. Birn, \& E. R. Priest Birn, J., Galsgaard, K., Hesse, M., et al. 2005, Geophys. Res. Lett., 32, 6105 Brown, J. C. 1971, Sol. Phys., 18, 489

Dmitruk, P., \& Matthaeus, W. H. 2006, J. Geophys. Res. Space Phys., 111, 12110 Dmitruk, P., Matthaeus, W. H., Seenu, N., \& Brown, M. R. 2003, ApJ, 597, L81 Dmitruk, P., Matthaeus, W. H., \& Seenu, N. 2004, ApJ, 617, 667

Holman, G. D. 2003, ApJ, 586, 606

Hoyng, P., Duijveman, A., Machado, M. E., et al. 1981, ApJ, 246, L155+ Isobe, H., Yokoyama, T., Shimojo, M., et al. 2002, ApJ, 566, 528

Jing, J., Lee, J., Liu, C., Gary, D. E., \& Wang, H. 2007, ApJ, 664, L127

Krucker, S., Hurford, G. J., MacKinnon, A. L., Shih, A. Y., \& Lin, R. P. 2008, ApJ, 678, L63

Lin, R. P. 2008, in ed. G. Li, Q. Hu, O. Verkhoglyadova, G. P. Zank, R. P. Lin, \& J. Luhmann, Am. Inst. Phys. Conf. Ser., 1039, 52

Lin, R. P., \& Hudson, H. S. 1976, Sol. Phys., 50, 153

Lin, R. P., \& Rhessi Team. 2002, in Solar Variability: From Core to Outer Frontiers, ed. A. Wilson, ESA SP, 506, 1035

Lin, R. P., Krucker, S., Hurford, G. J., et al. 2003, ApJ, 595, L69

Liu, C., \& Wang, H. 2009, ApJ, 696, L27

Liu, C., Lee, J., Jing, J., Gary, D. E., \& Wang, H. 2008a, ApJ, 672, L69

Liu, W., Petrosian, V., Dennis, B. R., \& Jiang, Y. W. 2008b, ApJ, 676, 704

Masuda, S., Kosugi, T., Hara, H., Tsuneta, S., \& Ogawara, Y. 1994, Nature, 371, 495

Moreno-Insertis, F., Galsgaard, K., \& Ugarte-Urra, I. 2008, ApJ, 673, L211

Nordlund, A., \& Galsgaard, K. 1995, A 3D MHD Code for Parallel Computers, Tech. rep., Astronomical Observatory, Copenhagen University

Northrop, T. G. 1964, Am. J. Phys., 32, 807

Petschek, H. E. 1964, NASA SP, 50, 425

Press, W. H., Teukolsky, S. A., Vetterling, W. T., \& Flannery, B. P. 1992, Numerical recipes in FORTRAN. The art of scientific computing (Cambridge: University Press), 2nd edn.

Priest, E., \& Forbes, T. 2000, Magnetic Reconnection, ed. E. Priest, \& T. Forbes Shibata, K., Nakamura, T., Matsumoto, T., et al. 2007, Science, 318, 1591

Turkmani, R., Cargill, P. J., Galsgaard, K., Vlahos, L., \& Isliker, H. 2006, A\&A, 449, 749 